\title{
Fifth Anniversary Editorial
}

This first 2015 issue of Cardiovascular Engineering and Technology (CVET) is a milestone for our journal. In its sixth volume, CVET is now five years young. The journal was launched by BMES in 2010 to serve the cardiovascular engineering community: academia, industry and clinicians. We owe a debt of gratitude to the many people who have supported us along the way, as well as to the many contributors who have submitted their exceptional work to our journal.

Today our journal is shifting from the position of a new publication to a journal with a solid reputation, aiming to be the "one stop" journal for cardiovascular engineering and technology and translational research dissemination. As such, we believe that the scientific and editorial character and quality of our journal merit its inclusion in PubMed Central (PMC) and we have now submitted our application. In addition, we have also applied for our journal evaluation and selection at Electronic Social and Science Citation Index (ESSCI).

On average, we publish roughly 100 pages (i.e. approximately 10 articles) per issue. To name a few more statistics, the average number of days between the date the manuscript was received and the first reviewer was invited was 7.5 in the previous year of the fifth volume. In the same year, the average number of days between the date the manuscript was received and the first decision was made is 48 . As it can be seen in the graphs below, this number has increased, compared to the fourth volume, due to the new policy under which we often have to return the manuscript to the authors to include the conflict of interest and statements of human and animal studies as one of the requirements for the PMC and ESSCI applications.

In addition to the PMC and ESSCI listings, our vision for the journal also includes: producing more special issues; participating in active article solicitation; increasing journal marketing; expanding the area of expertise of the editorial board; creating partnerships with conferences; and engaging industry and clinical experts in the publication and review process.

As Founding Editor of CVET, I have just begun my second term and over the next 3 years I will lead the journal to successfully reach its vision. In keeping with CVET's mission, I am also excited to announce our
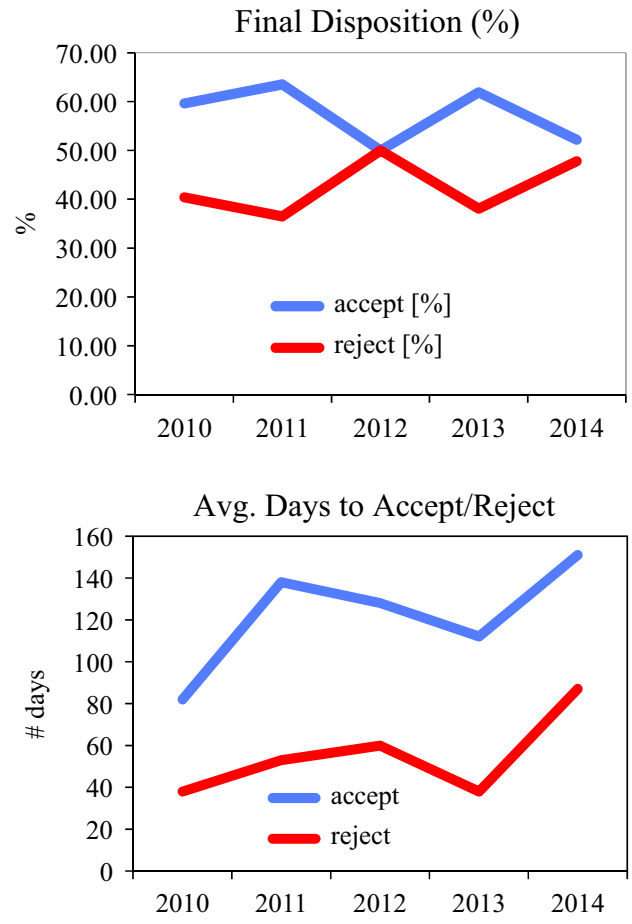

next special issue in June 2015 which will include approximately 10 articles/100 pages dedicated to mitral valve mechanics, with Dr. Karyn S. Kunzelman as the guest editor.

A special debt of gratitude is owed to our referees and members of the editorial board whose work maintains the scientific standards of the journal without which we could never reach this threshold. With the continued support of the cardiovascular engineering and technology community, we hope to continue to deliver a wide spectrum of stimulating papers which will inspire our readers and contribute to the progress in biomedical academia; medical device, diagnostic and imaging industries; and clinical cardiovascular medicine and surgery.

Ajit P. Yoganathan

Editor-in-Chief

Atlanta, Georgia, USA

Electronic mail: ajit.yoganathan@bme.gatech.edu 